\title{
Controlling Hierarchical Self-Assembly in Supramolecular Tailed-Dendron Systems
}

\author{
Nathalie Merlet-Lacroix ${ }^{\dagger}$ Jingyi Rao, ${ }^{\star}$ Afang Zhang, ${ }^{\ddagger}$ A. Dieter Schlüter, \\ Sreenath Bolisetty, ${ }^{*}$ Janne Ruokolainen, ${ }^{\S}$ and Raffaele Mezzenga ${ }^{*}, *, \|$ \\ ${ }^{\dagger}$ Department of Physics and Fribourg Centre for Nanomaterials, University of Fribourg, Ch. Du Musée 3, \\ CH-1700 Fribourg, Switzerland, ${ }^{*}$ Laboratory for Polymer Chemistry, Swiss Federal Institute of Technology, \\ Department of Materials, ETH-Zurich, HCI J541, CH-8093 Zurich, Switzerland, ${ }^{\S}$ Helsinki University of \\ Technology, Physics Laboratory, Helsinki 02015, Finland, and "Food and Soft Materials Science, \\ Institute of Food, Nutrition and Health, ETH Zurich, CH-8092 Zurich, Switzerland
}

\begin{abstract}
We study the self-assembly of a dendritic macromolecular system formed by a secondgeneration dendron with $\mathrm{pH}$-responsive end groups and with a polymer chain emanating from its focal point, typically referred to as dendron-coil system. We use supramolecular ionic interactions to attach to the periphery of the dendrons sulfate-terminated alkyl tails of various lengths. The resulting ionic complexes have a molecular architecture similar to a four-arm dendritic pitchfork with varying arms and holder lengths. The bulk morphologies observed by small-angle X-ray scattering (SAXS) and transmission electron microscopy (TEM) show thermodynamically stable, hierarchical "inverted" hexagonal or lamellar structures. In addition, for a specific range of volume fractions, we show order-to-order transitions associated with the melting of the crystalline alkyl tails. The structural models for the molecular packing emerging from TEM and SAXS analysis are benchmarked to available self-consistent field theories (SCFTs) developed for identical systems and experiments and theoretical predictions are found in perfect agreement. With respect to our previous work on inverted dendron and dendrimer-surfactant self-assembled morphologies (Mezzenga et al. Soft Matter. 2009, 5, 92-97), the present findings show that keeping the same dendritic molecular architecture but adding a polymer chain emanating from the focal point enables the scale up of the structural organization from the liquid crystalline length scale $\left(10^{0} \mathrm{~nm}\right)$ to the block copolymer length scale $\left(10^{1} \mathrm{~nm}\right)$ while preserving the inverted unconventional morphologies. Because the length of the holder and the arms of the dendritic pitchfork can be finely tuned, these systems offer new possibilities in the design of nanostructured organic materials and their use in templating applications.
\end{abstract}

\section{Introduction}

Supramolecular chemistry has gained remarkable attention in the last 20 years as a very powerful technique to design complex macromolecular systems based on noncovalent bonds. ${ }^{1,2}$ The self-assembly of these materials offers nearly limitless possibilities because the architecture of the macromolecular template, the nature of the supramolecular interactions, as well as the diversity of the ligands can be finely controlled to tune the morphologies and properties of the final complexes formed..$^{2-6}$

Dendrons, dendrimers, and dendronized polymers have emerged as unmatched building blocks for supramolecular polymer science. $^{7-26}$ These highly branched molecules are of unique interest because their typical size and the number of peripheral groups, which participate in supramolecular interactions, can be designed by simply changing their generation. ${ }^{7}$ These macromolecular templates have an intrinsic "concave" curvature at the dendritic surface with respect to the focal point, which has led previous studies on the self-assembly of dendrons and dendrimerbased systems to the assumption that this concave dendrimer/ pendant chain interface is maintained in the bulk structures. ${ }^{8-24}$ We have, however, recently shown that in columnar liquid crystalline phases based on dendronized polymers, dendrons,

*Corresponding author. E-mail: raffaele.mezzenga@agrl.ethz.ch. Tel: +41 446329140 . or dendrimers ionically complexed to alkyl tails, "inverted" topological configurations (with the pendant chains inside the columns) are possible in the solid state, provided that the volume fractions of the side chains do not substantially exceed the majority component. ${ }^{25,26}$ These results have some surprising connotation in the case of the dendrons and particularly dendrimers, in which the intrinsic natural curvature is more pronounced than that of corresponding dendronized polymers. Furthermore, self-consistent field theory simulations (SCFTs) support the occurrence of inverted configurations as thermodynamically stable structures for these complexes at the volume fractions investigated. ${ }^{26}$ These exciting results open new routes to the design of porous nanotemplates if the surfactants restricted within the columns in the hexagonal or rectangular packing can be removed by exploiting their noncovalent bindings to the dendrons. ${ }^{27}$

To understand to which extent this "inverted" self-assembly scheme is maintained and to control the resulting structural properties of the nanotemplates, we have used the same secondgeneration dendron as in ref 26 but with a polymer chain emanating out of the focal point. The polymerization degree of this polymer chain and thus its volume fraction can be maintained as a tunable parameter. We will demonstrate hereafter that this new molecular parameter is in fact a key variable in determining the final self-assembled morphologies. The secondgeneration dendron, which exhibits as a consequence a reduced 

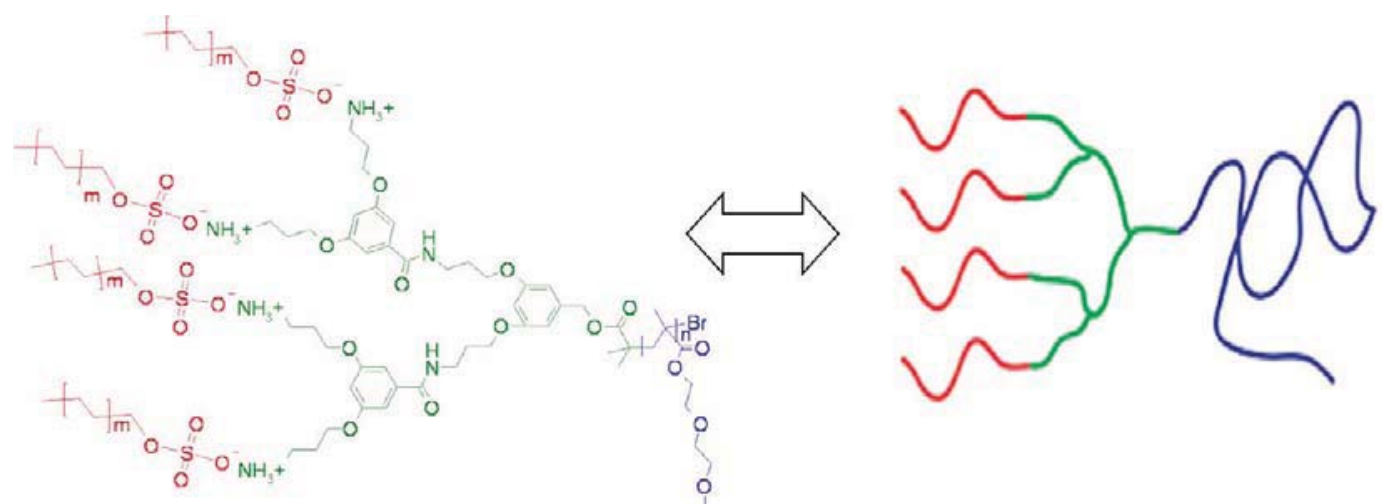

Figure 1. Schematic drawings of the complexes used in this study. A second-generation dendron (G2, green) is covalently attached to a PMeDEG polymer tail (blue) where the number of repeating units $(n)$ is either 26 or 33 . The surfactants (red) that interact with $\mathrm{NH}_{3}^{+}$via ionic bonding have 12 or 18 carbons ( $m=5$ or 8 ). On the left-hand side, the chemical formulas of the different species are shown, whereas on the right side of the Figure, a more schematic drawing of the complexes is presented.

degree of freedom, is then ionically complexed to sulfate-terminated alkyl tail surfactants of varying hydrocarbon lengths, and the resulting self-assembled structures in bulk are studied by SAXS and TEM.

Prevailing morphologies are identified as core-shell cylinders and hierarchically organized lamellae, both exhibiting long-range order. Temperature-dependent SAXS experiments are used to demonstrate unambiguously that the morphologies are thermodynamically stable. In addition, order-to-order transitions from hierarchical lamellae to core-shell hexagonal cylinders are observed in correspondence of the melting of surfactant crystallinity. Results are rationalized in terms of SCFTs available for identical dendritic pitchfork-like systems, ${ }^{28}$ and we compare the results with the liquid crystalline structures observed without the presence of the linear polymer tail emanating from the focal point of the dendrons, thus highlighting the importance of a chain extension of the dendrons for the design of hierarchically organized structures with a high degree of order.

\section{Experimental Section}

Material and Complex Preparation. Second-generation dendrons were synthesized according to known procedures. ${ }^{29}$ The covalent bonding of the polymer tail at the focal point of the dendron was realized via atom transfer radical polymerization of methoxy diethylene glycol methacrylate (MeDEG), and the polymerization degree of this polymer tail was designed to keep its mass relevant to that of the dendron. (Detailed information is available in the Supporting Information.) After deprotection with trifluoroacetic acid, the dendron-terminated polymers carry four ammonium end groups. NMR was carried on the deprotected systems, and no degradation was observed. To retain the positive charges of the end functional groups of the dendrons, the macromolecular templates were dissolved in acidic water $(5<\mathrm{pH}<6$, adjusted with $\mathrm{HCl})$. A separated solution of water and sulphated alkyl tails was prepared at identical $\mathrm{pH}$. Ionic complexes were prepared by adding dropwise to the alkyl sulfate solution the equivalent molar mass of the dendritic solution under continuous stirring. For complexation with $\mathrm{C}_{18} \mathrm{SO}_{4}{ }^{-}$due to its lower solubility in water, $20 \%$ isopropanol was added, and the surfactant solution was heated to $40{ }^{\circ} \mathrm{C}$ for a few minutes. In each case, upon addition of the polymer, the solutions became turbid, indicating the formation of stoichiometric complexes, ${ }^{11}$ as we have already observed and characterized in our previous studies on dendritic polyelectrolytes-counter-charged surfactants ionic complexes. ${ }^{25-27} \mathrm{Com}-$ plexes were collected after centrifugation and removal of the water and then dried at $50^{\circ} \mathrm{C}$ for 1 day under low vacuum. The annealing of the samples was performed under high vacuum $\left(<10^{-7} \mathrm{mBar}\right)$ at temperatures above the glass transition of the
G2-PMeDEG polymers and maintained systematically between the melting temperature of the alkyl tails and the orderdisorder transition of the complexes (determined by SAXS measurements at different temperatures).

Small-Angle X-ray Scattering (SAXS). Simultaneous smalland wide-angle X-ray scattering (SWAXS) experiments were performed on a SAXSess instrument (Anton Paar) using a $\mathrm{Cu}$ $\mathrm{K} \alpha(\lambda=0.1542 \mathrm{~nm}) \mathrm{X}$-ray source and a slit camera configuration. A highly sensitive SWAXS imaging plate at $263.3 \mathrm{~nm}$ from the sample is used to collect the signal under vacuum. The temperature of the sample is controlled by a homemade regulator between 20 and $200{ }^{\circ} \mathrm{C}$. Previously dried powder samples were placed in between two mica foils in the sample holder, and diffraction data were acquired for $1 \mathrm{~h}$. For temperature-dependent measurements, each diffractogram was acquired after $30 \mathrm{~min}$ of equilibration at isothermal temperature. Measurements were repeated at least twice on different batches of the same complexes and were shown to be perfectly reproducible.

Transmission Electron Microscopy and cryo-TEM. TEM was carried out using a Jeol JEM-3200FSC field emission microscope operating at $300 \mathrm{kV}$ voltage. The images were taken in bright field mode and using zero loss energy filtering (omega type) with the slit width of $20 \mathrm{eV}$. Micrographs were recorded using Gatan Ultrascan 4000 CCD camera. Specimen temperature was maintained at $-187^{\circ} \mathrm{C}$ during the imaging using liquid nitrogen. For structures constituted by core-shell columnar hexagonal cylinders, the presence of PMeDEG in the continuous phase made challenging the use of TEM, as sections were found to be very unstable to the electron beam. In a further effort to stabilize these sections against electron beam, Heliumcooled cryoTEM was also employed, in which sections maintained at $18 \mathrm{~K}$ did exhibit improved stability. Thin sections $(\sim 70 \mathrm{~nm})$ were cut at $-60^{\circ} \mathrm{C}$ by Leica Ultracut UTC microtome using a $25^{\circ}$ Diatome diamond knife. The sections were collected on 300 mesh lacey carbon grids. The samples were stained in vapor of $0.5 \% \mathrm{RuO}_{4}$-stabilized aqueous solution (Electron Microscopy Sciences) for $1 \mathrm{~min}$. $\mathrm{RuO}_{4}$ selectively stains the aromatic rings present in the dendron molecule of the complexes, which confers them a dark contrast in the TEM micrographs. In addition, partial $\mathrm{RuO}_{4}$-staining of the PMeDEG polymers confers a gray color to domains made thereof. Structure periodicities and lattice parameters were calculated from Fourier transformed micrographs.

\section{Results and Discussion}

Figure 1 provides a scheme of the resulting supramolecular complexes, whereas Table 1 gives the volume fractions of the individual molecular segments. ${ }^{25}$ The number of repeating units in the polymer chain was 26 or 33 , and the surfactants employed 
in the complexation were $\mathrm{C}_{12} \mathrm{SO}_{4}{ }^{-}$and $\mathrm{C}_{18} \mathrm{SO}_{4}{ }^{-}$. As a consequence, four complexes have been prepared and studied: G2$\mathrm{PMeDEG}_{26^{-}} \mathrm{C}_{12} \mathrm{SO}_{4}, \mathrm{G} 2-\mathrm{PMeDEG}_{26}-\mathrm{C}_{18} \mathrm{SO}_{4}, \mathrm{G} 2-\mathrm{PMeDEG}_{33^{-}}$ $\mathrm{C}_{12} \mathrm{SO}_{4}$, and $\mathrm{G} 2-\mathrm{PMeDEG}_{33}-\mathrm{C}_{18} \mathrm{SO}_{4}$.

Figure 2a presents the SAXS spectra acquired at different temperatures for the complex G2-PMeDEG ${ }_{26}-\mathrm{C}_{12} \mathrm{SO}_{4}$. The very same structure consisting of several Bragg reflections is observed at all temperatures below $90{ }^{\circ} \mathrm{C}$. At $90^{\circ} \mathrm{C}$, the Bragg reflections disappear and the diffractogram evolves into a single broad peak scattering curve, characteristic of disordered systems exhibiting a correlation peak ${ }^{30}$ and indicating that the order-disorder transition $\left(T_{\mathrm{ODT}}\right)$ has been passed. When the sample is cooled back to room temperature (upper curve), the same Bragg peaks appear at identical positions, and the peaks are better resolved, indicating that the structure is thermodynamically stable and that thermal annealing increases the degree of order. A closer inspection of the position of the peaks shows that they are spaced as $q_{1}: q_{2}: q_{3}: q_{4}: q_{5}: q_{6}=1: \sqrt{ } 3: 2: \sqrt{ } 7: 3: \sqrt{ } 12$, which is consistent with a columnar hexagonal phase with lattice period of $11 \mathrm{~nm}$ exhibiting as many as six Bragg reflections and thus characterized by a very long-range order. Although the Bragg reflections leave no space to doubts on the columnar hexagonal lattice organization, analysis of the form factor emerging from Figure 2a suggests a nontrivial structure. Indeed, for example, the relative heights of the first four peaks are nearly the same, whereas the intensity of reflections in a simple columnar hexagonal phase should decrease strongly with the order of the Bragg reflection. We infer here that this is due to the form factor of the cylinders. Figure $2 b$ shows the corresponding TEM micrograph where contrast is achieved by $\mathrm{RuO}_{4}$, which selectively stains the aromatic rings of the dendrons. The very low contrast does not allow us to assess the morphology conclusively, although a hint of parallel cylinders can be spotted on the top left corner in the Figure. Generally speaking, the TEM imaging of the hexagonal structures turned out to be extremely

Table 1. Volume Fractions of the Different Species in the Four Supramolecular Complexes ${ }^{a}$

\begin{tabular}{lclcc}
\hline & $\varphi_{\text {surfactant }}$ & $\varphi_{\mathrm{G} 2}$ & $\varphi_{\text {surfactant }+\mathrm{G} 2}$ & $\varphi_{\text {polymer }}$ \\
\hline $\mathrm{G}-\mathrm{PMeDEG}_{26}-\mathrm{C}_{12} \mathrm{SO}_{4}$ & 0.151 & 0.085 & 0.236 & 0.764 \\
$\mathrm{G}-\mathrm{PMeDEG}_{26}-\mathrm{C}_{18} \mathrm{SO}_{4}$ & 0.180 & 0.082 & 0.262 & 0.738 \\
$\mathrm{G}-\mathrm{PMeDEG}_{33}-\mathrm{C}_{12} \mathrm{SO}_{4}$ & 0.125 & 0.07 & 0.195 & 0.805 \\
$\mathrm{G}-\mathrm{PMeDEG}_{33}-\mathrm{C}_{18} \mathrm{SO}_{4}$ & 0.150 & 0.068 & 0.218 & 0.782 \\
${ }^{a}$ Respective densities & $\left(\mathrm{g} / \mathrm{cm}^{3}\right)$ measured by a helium pycnometer: \\
$d_{\mathrm{C} 12}=1.06, d_{\mathrm{C} 18}=1.16, d_{\mathrm{G} 2}=1.52, d_{\text {polymer }}=0.993$.
\end{tabular}

difficult in each case, and this is believed to be the consequence of two main factors: (i) the PMeDEG polymer is a very low $T_{\mathrm{g}}$ polymer $\left(-27.1^{\circ} \mathrm{C}\right)$, which makes the sample very fluid, and thus the sections are particularly unstable to staining and electron beam imaging, and (ii) the PMeDEG polymer is derived from polymethylmethacrylate backbones, which notoriously undergo degradation upon TEM electron beam exposure. Whereas this directly supports the fact that the low $T_{\mathrm{g}}$ PMeDEG polymer forms the continuous phase, more convincing TEM images of cylinders will be presented later.

On the basis of the chemical structure of the supramolecular complex, the calculated volume fractions (Table 1), and the X-ray scattering profile, we suggest that the cylinders adopt a core-shell structure, as schematically drawn in Figure 2c, with the surfactants in the core of the cylinders (volume fraction below 0.2), the dendrons in the shells, and the polymer filling the space between the cylinders (highest volume fraction).

To reveal in the real space the structure of the hexagonally packed core-shell cylinders further, helium-cooled cryogenic TEM was also employed. This nontrivial technique, borrowed directly from biological sciences, has the benefit to stabilize further against beam damage samples having low- $T_{\mathrm{g}}$ and methacrylate-based backbones, such as those used in the present case to design the hexagonal structures. Figure 3 shows a composite cryoTEM image of sample G2-PMeDEG $26^{-} \mathrm{C}_{12} \mathrm{SO}_{4}$. In part a, a lower magnification of the cross-sectional cut of the columnar core-shell cylinders is illustrated. A hierarchical structure of the cylinders with a white inner spot, a dark corona, and a gray continuous phase can now be clearly resolved. The closest distance among the white inner spots is $10.8 \mathrm{~nm}$, which is in excellent agreement with the $11.0 \mathrm{~nm}$ measured by SAXS analysis. Figure $3 \mathrm{~b}$ shows a zoom of the cross-sectional structure of the cylinders, revealing additional details of the close packing of the core-shell cylinders, whereas Figure $3 \mathrm{c}$ shows the longitudinal projection of the cylinders, again constituted by a white inner core, dark intermediate domains, and gray domains in between.

Figure 4a presents the SAXS diffractogram measured for the complex G2-PMeDEG ${ }_{26}-\mathrm{C}_{18} \mathrm{SO}_{4}$ at room temperature. The six peaks at low $q$, spaced as $q_{1}: q_{2}: q_{3}: q_{4}: q_{5}: q_{6}=1: 2: 3: 4: 5: 6$, are typical of a very long-range ordered lamellar phase with a period of $13 \mathrm{~nm}$. The large $q$ region is also shown to highlight the presence of a peak around $16.7 \mathrm{~nm}^{-1}$ due to the crystallinity of the surfactant chains. SAXS measurements performed at different

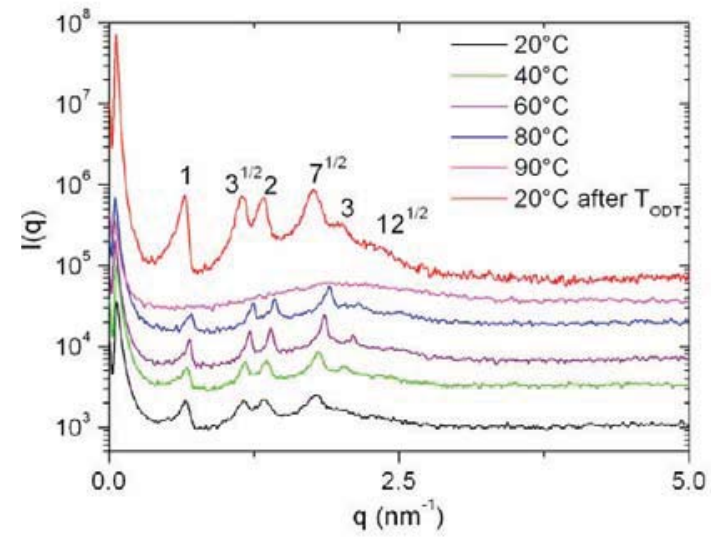

(a)

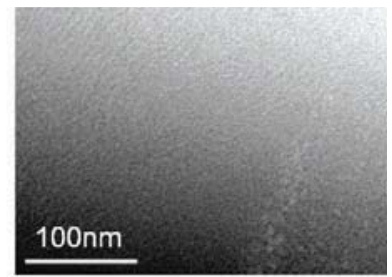

(b)

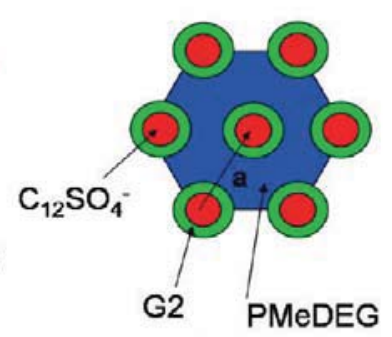

(c)

Figure 2. (a) SAXS diffractogram at different temperatures, (b) TEM picture at room temperature, and (c) scheme of the deducted morphology for complex G2-PMeDEG $26-\mathrm{C}_{12} \mathrm{SO}_{4}$. 

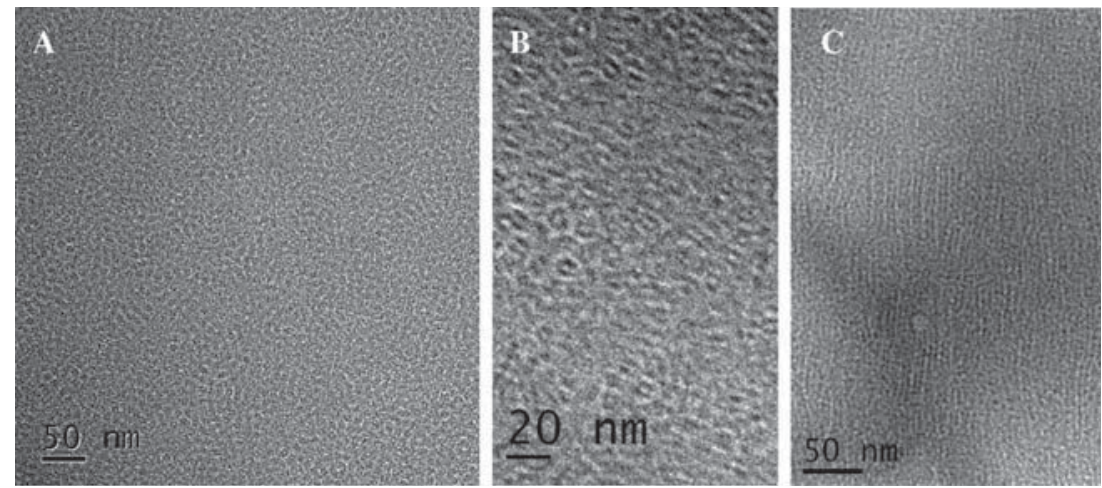

Figure 3. Helium-cooled cryogenic transmission electron micrographs for G2-PMeDEG ${ }_{26}-\mathrm{C}_{12} \mathrm{SO}_{4}$ complexes. (a) Low magnification of the crosssectional cut of the columnar core-shell cylinders, revealing core-shell structures with white inner spot, dark corona, and a gray continuous phase. The closest neighbor distance between white inner spots is $10.8 \mathrm{~nm}$. (b) Zoom of the cross-sectional structure of the cylinders. (c) Longitudinal projection of the cylinders, characterized by a white inner core, dark intermediate domains, followed by gray domains.

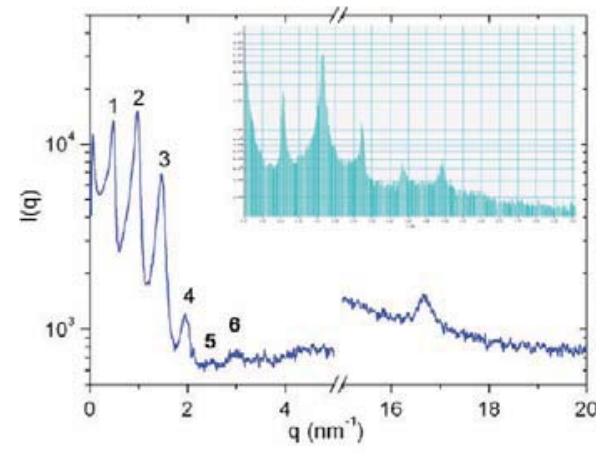

(a)

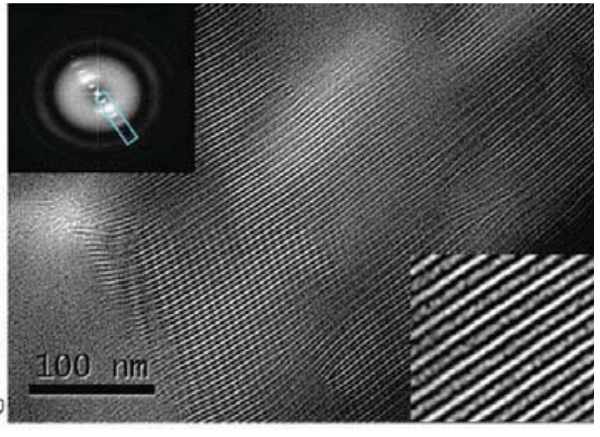

(b)

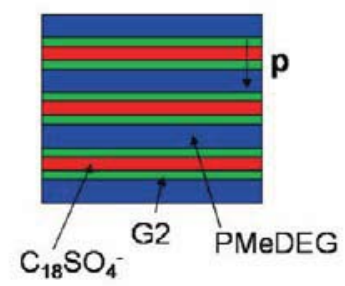

(c)

Figure 4. (a) SAXS diffractogram and (b) TEM picture at room temperature for complex $\mathrm{G} 2-\mathrm{PMeDEG}_{26}-\mathrm{C}_{18} \mathrm{SO}_{4}$. On the lower right corner of b, a zoom of a specific area is presented. On the upper left corner of part $b$, the Fourier transform of the TEM picture is shown and its integration is presented in the inset of part a. The deducted morphology is schematically represented (c).

temperatures prove that the crystallinity of the small chains melts at $90^{\circ} \mathrm{C}$, but the lamellar morphology remains up to $110^{\circ} \mathrm{C}$. At $120^{\circ} \mathrm{C}$, the $T_{\mathrm{ODT}}$ is passed and the system enters the disordered region. When the sample is cooled to room temperature, the morphology is found to be lamellar again, proving that the structure is thermodynamically stable. Yet, the form factor of the lamellar phase is unusual with the multiple Bragg reflections of rather uncommon high intensities and, in particular, with the second peak higher that the first Bragg reflection; we will show below that this effect is due to a hierarchical packing of the lamellae.

Figure $4 \mathrm{~b}$ shows the TEM picture of the same complex, again stained with $\mathrm{RuO}_{4}$. Differently from the core-shell hexagonal phases, this complex exhibits a solid-like behavior because the PMeDEG domains now occupy 2D layers in contrast with the continuous 3D space-filling domains of the hexagonal phase. This trend, together with the lower volume fraction of PMeDEG, greatly improves the stability of the sections to staining and electron beam damage. The contrast between the different domains is very good, although the ratio of volume fraction of the different blocks cannot be precisely measured from the TEM because the images are acquired slightly out of focus to enhance the contrast between the different domains. Yet, the period of the lamellae can be measured and is in perfect agreement with the SAXS data. In the lower right inset of Figure $4 \mathrm{~b}$, the hierarchical organization of lamellae can be clearly observed, with the black domains corresponding to the stained dendrons, the white domains corresponding to the unstained surfactants, and the gray domains corresponding to the partially stained polymer tails. $\left(\mathrm{RuO}_{4}\right.$ can mark ethers and phenyl groups, but the staining of phenyl groups occurs more quickly and efficiently, which explains the black color for dendrons and the gray for polymers.) The organization of the G2-PMeDEG ${ }_{26}-\mathrm{C}_{18} \mathrm{SO}_{4}$ complexes is sketched in Figure 4c. These findings demonstrate that the surfactant/dendron/polymer are incompatible, which further supports the core-shell cylinders arguments given for the G2-PMeDEG ${ }_{26}-\mathrm{C}_{12} \mathrm{SO}_{4}$ complex.

As an additional comparison between TEM results and diffraction data, a fast Fourier transform (FFT) of the TEM picture is shown as an inset on the upper left corner of Figure $4 \mathrm{~b}$. 


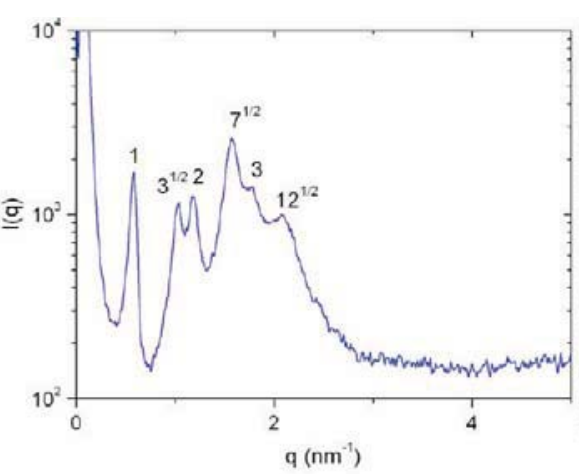

(a)

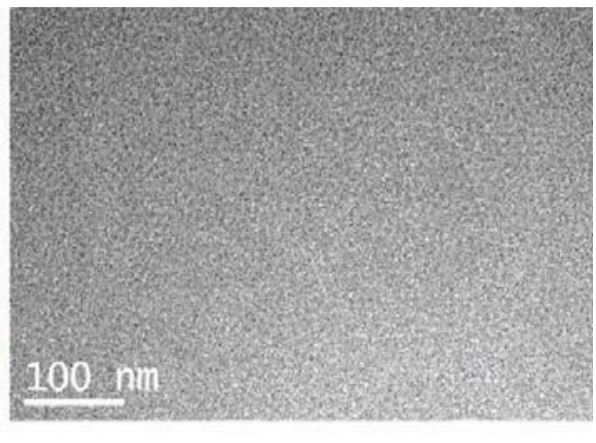

(b)

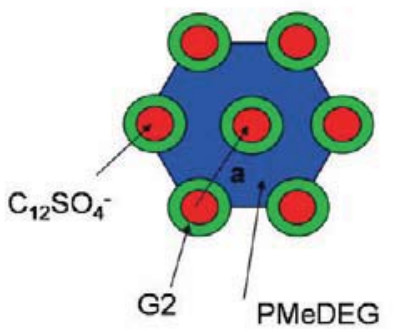

(c)

Figure 5. (a) SAXS diffractogram and (b) TEM picture for complex G2-PMeDEG $33-\mathrm{C}_{12} \mathrm{SO}_{4}$ at room temperature. The deducted morphology is schematically drawn in (c).

The intensity along the highlighted rectangular zone of the FFT has been integrated, and the integration profile is presented in the inset of Figure $4 \mathrm{a}$. The similarity with the scattering profile is remarkable, with high-order Bragg reflections of high intensities and with the second reflection stronger than the first. This clearly demonstrates that the unconventional form factor of the lamellar phase observed by SAXS arises from the hierarchical organization of lamellae.

Hierarchical structures have already been observed for block copolymers in which microphase segregation between two incompatible blocks can be combined with supramolecular attachment of side chains, or mesogens, or surfactants to one block. ${ }^{5,6,31-34}$ In these systems, self-assembly is typically organized on two length scales: that of the side chains and that of the polymers. The most frequently observed structure at the lowest length scale is lamellar, with side-chain layers orientated orthogonally to the higher periodicity flat interfaces. Additional topological configurations have been recently achieved by using more complex systems in which one of the blocks is a dendronized polymer capable of participating in supramolecular interactions. ${ }^{35}$ These supramolecular structures can exhibit columnar phases with rectangular, square, and hexagonal lattice on the small length scale as well as lamellae-in-lamellae, but the orientation of the surfactant domains (columnar or lamellar) is still orthogonal to the block copolymer interfaces because of the topological connectivity among the different chemical components. ${ }^{35}$

Experimental and theoretical works with multiblock terpolymers (C- $b-(\mathrm{B}-b-\mathrm{A})_{\mathrm{m}}-b$-B- $b$-C) have shown hierarchical lamellae in lamellae structures where the lamellae are parallel ${ }^{36-39}$ or orthogonal to each other, ${ }^{40,41}$ but to achieve this self-assembly scheme, very complex chemical structures and high molecular weights have to be employed, which enable together high degrees of segregation. As a result, the longer periodicity for these systems often approaches $100 \mathrm{~nm}$. Therefore, to our knowledge, the complexes disclosed in this study are the first supramolecular system capable of self-assembling into both core-shell hexagonal cylinders and parallel hierarchical lamellae-in-lamellae, and both on length scales of ca. $10 \mathrm{~nm}$, that is, 1 order of magnitude lower than what previously reported in literature.
Figure 5a presents the SAXS diffractogram of the complex G2$\mathrm{PMeDEG}_{33}-\mathrm{C}_{12} \mathrm{SO}_{4}$. As many as six peaks are present, spaced as $q_{1}: q_{2}: q_{3}: q_{4}: q_{5}: q_{6}=1: \sqrt{ } 3: 2: \sqrt{ } 7: 3: \sqrt{ } 12$, which, again, is typical of a very well-ordered columnar hexagonal structure with a period of $12.5 \mathrm{~nm}$. Once more, the SAXS form factor and the heights of the individual peaks suggest a nontrivial hexagonal packing, which can be interpreted in terms of a core-shell structure of the cylinders, resulting from the incompatibility between the dendron, the linear polymer, and surfactants-rich domains. Although the contrast achievable with $\mathrm{RuO}_{4}$ staining in TEM micrographs is again weak (Figure 5b), a hexagonal packing can be recognized for the structure. Cryogenic TEM did not significantly increase the quality of the images, possibly because of the higher volume fraction of the PMeDEG in $\mathrm{G}_{2}-\mathrm{PMeDEG}_{33^{-}}$ $\mathrm{C}_{12} \mathrm{SO}_{4}$ as compared with $\mathrm{G} 2-\mathrm{PMeDEG}_{26}-\mathrm{C}_{12} \mathrm{SO}_{4}$. The resulting morphology of this complex is schematically shown in Figure $5 \mathrm{c}$ with the surfactants occupying the core of the cylinders, the dendrons occupying the shell, and the polymer filling the space in between the cylinders. This morphology does not vary significantly with temperature, as studied by SAXS, and is thermodynamically stable up to $100^{\circ} \mathrm{C}$, temperature at which the $T_{\mathrm{ODT}}$ is reached. Again the morphology recovered at room temperature after going above the $T_{\mathrm{ODT}}$ is identical, indicating thermodynamically stable structures.

To support the core-shell hexagonally packed cylinders morphologies further, we have fitted the experimental SAXS profile of the G2-PMeDEG ${ }_{26}-\mathrm{C}_{12} \mathrm{SO}_{4}$ and $\mathrm{G} 2-\mathrm{PMeDEG}_{33}-\mathrm{C}_{12} \mathrm{SO}_{4}$ samples with the expected theoretical curves.

The expected diffracted X-ray intensity for an ensemble of equal scatterers is given by $I(q)=n P(q) S(q)$, in which $n$ is the number of scattering objects, $P(q)$ is the form factor of the scatterers, and $S(q)$ is the structure factor of the geometrical organization of their centers of mass. Because we work in arbitrary units for the diffracted intensity, the number of scattering objects, $n$, can be omitted from the fitting, and the expected diffracted intensity is $I(q) \cong P(q) S(q)$, in which $P(q)$ is the form factor of core-shell cylinders and $S(q)$ is the structure factor of a columnar hexagonal lattice. Figure $6 \mathrm{a}, \mathrm{b}$ compares the experimental profile of the SAXS diffractograms of $\mathrm{G} 2-\mathrm{PMeDEG}_{33}-\mathrm{C}_{12} \mathrm{SO}_{4}$ and 

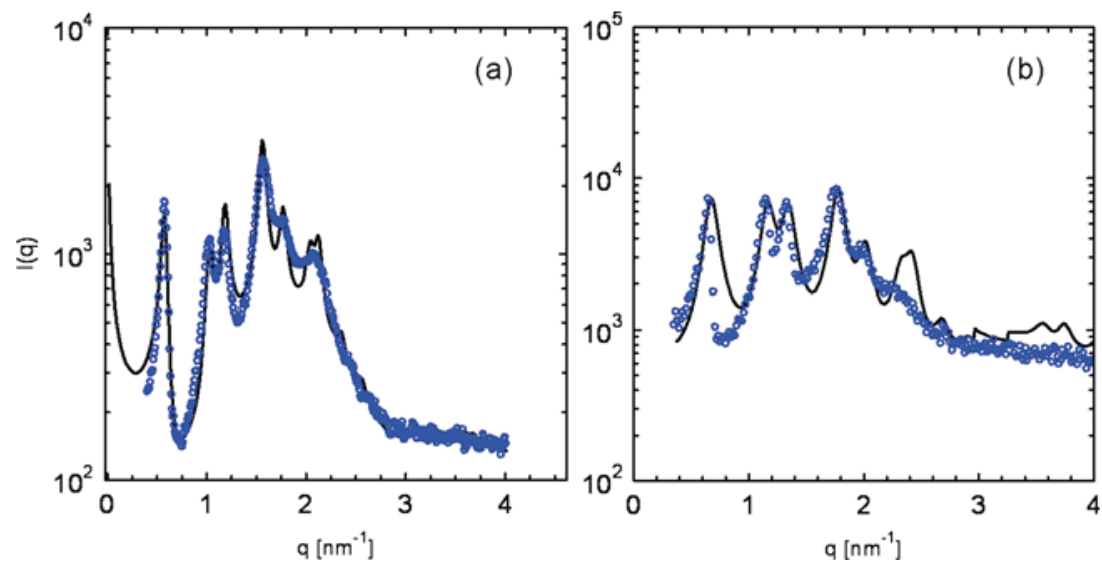

Figure 6. Fit of SAXS experimental data (open symbols) with the theoretical expected diffracted profile (full lines) for core shell cylinders hexagonally packed in a columnar lattice. (a) $\mathrm{G} 2-\mathrm{PMeDEG}_{33}-\mathrm{C}_{12} \mathrm{SO}_{4}$. The fit has been obtained by imposing a lattice parameter of $12.5 \mathrm{~nm}$, a core cylinder radius of $2.3 \mathrm{~nm}$, and a shell of $1.7 \mathrm{~nm}$, which are the values resulting directly from volume fractions considerations listed in Table 1. (b) $\mathrm{G}_{2}-\mathrm{PMeDEG}_{26}-\mathrm{C}_{12} \mathrm{SO}_{4}$. The fit has been obtained by imposing a lattice parameter of $11 \mathrm{~nm}$, a core cylinder radius of $2.2 \mathrm{~nm}$, and a shell of $1.5 \mathrm{~nm}$. Again, these values are not fitting parameters but result directly from volume fraction considerations listed in Table 1.

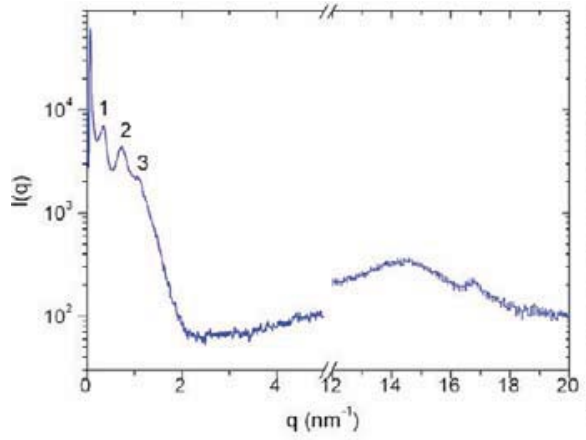

(a)

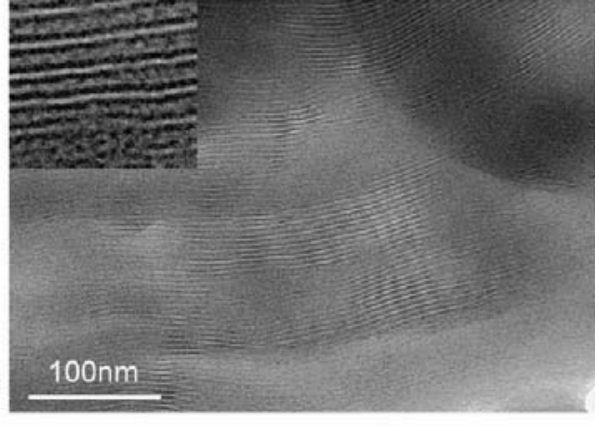

(b)

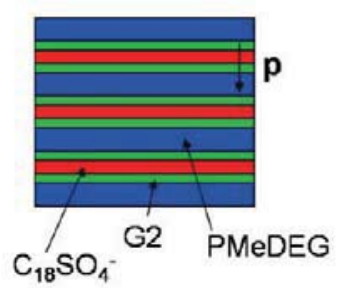

(c)

Figure 7. (a) SAXS diffractogram, (b) TEM picture at room temperature, and (c) scheme of morphology for complex $\mathrm{G}_{2}-\mathrm{PMeDEG}_{33}-\mathrm{C}_{18} \mathrm{SO}_{4}$ at room temperature. In the upper left corner of part $b$, a zoom of a specific area of the picture is shown.

G2-PMeDEG $26-\mathrm{C}_{12} \mathrm{SO}_{4}$ with the expected $I(q)$ for hexagonally packed core-shell cylinders with inhomogeneous shell, in which the surfactants form the internal cylinder core, the dendrons form the corona, and the polymers form the continuous phase. In both cases, the radius of the cylinders internal core and corona are those calculated on the basis of the volume fractions of the surfactant, dendron, and polymer reported in Table 1 so that the only fitting parameters are the ratios among the scattering length of the three species. Despite a few minor variations between the experimental data and predicted scattering, which are essentially due to the smearing of the slit camera used in the SAXS, a remarkable good agreement is obtained in both cases, which conclusively assesses the proposed morphology of coreshell hexagonally packed cylinders having the surfactants on their core, the dendrons in the corona, and polymer in the continuous phase.

Figure $7 \mathrm{a}$ presents the SAXS spectrum for the complex $\mathrm{G} 2-\mathrm{PMeDEG}_{33}-\mathrm{C}_{18} \mathrm{SO}_{4}$ at room temperature. The low $q$ peaks spaced as $q_{1}: q_{2}: q_{3}=1: 2: 3$ are typical of a lamellar phase with a period of $17 \mathrm{~nm}$ and an order somewhat lower than what was found for $\mathrm{G} 2-\mathrm{PMeDEG}_{26}-\mathrm{C}_{18} \mathrm{SO}_{4}$ complexes. The reflection at $16.5 \mathrm{~nm}^{-1}$ in the high $q$ range arises from the crystallinity of the surfactants. The TEM picture of this complex (Figure 7b) and the high magnification inset also show a lamellar morphology that is hierarchically ordered: the black domains correspond to the dendron phase, the gray domains corresponds to the polymer, and the white domains to the surfactant phase. The morphology of this complex is sketched in Figure 7c.

Although G2-PMeDEG $33-\mathrm{C}_{18} \mathrm{SO}_{4}$ and G2-PMeDEG ${ }_{26}-\mathrm{C}_{18} \mathrm{SO}_{4}$ have a similar structure, their thermotropic behavior is very different. The complex with the shortest polymer tail (G2-PMe$\mathrm{DEG}_{26}-\mathrm{C}_{18} \mathrm{SO}_{4}$ ), as described previously, maintains the lamellar morphology up to the order-disorder transition, even after the melting of the crystalline alkyl tails. On the contrary, for the G2-PMeDEG ${ }_{33}-\mathrm{C}_{18} \mathrm{SO}_{4}$ an order-to-order transition occurs with increasing temperature, as shown in Figure 8a. From room 


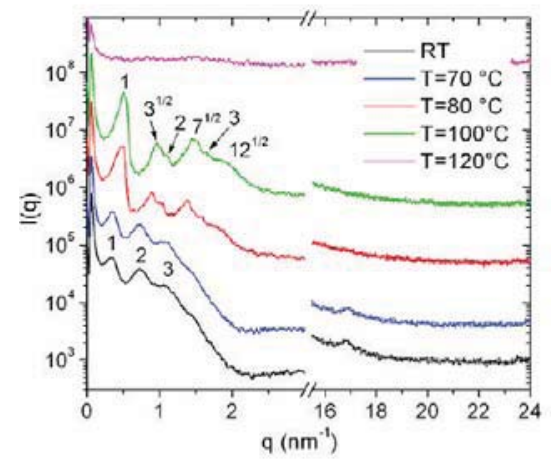

(a)

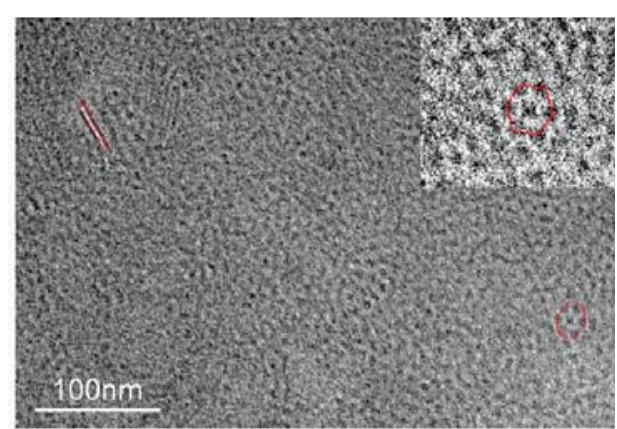

(c)

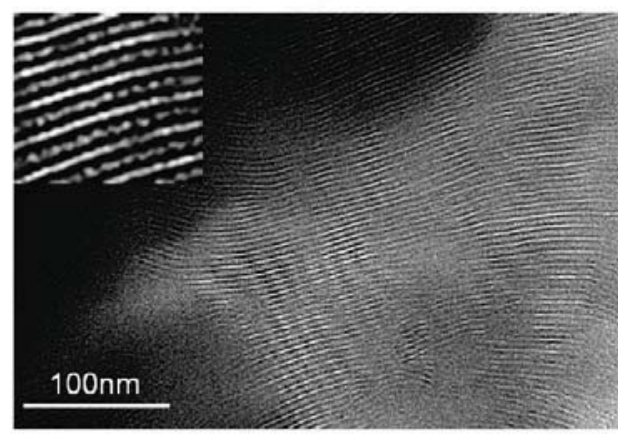

(b)

Figure 8. (a) SAXS diffractograms at different temperatures for the complex $\mathrm{G} 2-\mathrm{PMeDEG}_{33}-\mathrm{C}_{18} \mathrm{SO}_{4}$. We can observe an order-to-order transition when the temperature reaches $80^{\circ} \mathrm{C}$, followed by an order-to-disorder transition at a temperature of $120^{\circ} \mathrm{C}$. (b,c) TEM micrographs of the two different morphologies with insets showing a zoom of specific areas of interest.

temperature up to $70{ }^{\circ} \mathrm{C}$, the morphology is lamellar, and a crystallinity peak can clearly be observed at high $q$. Between 70 and $80^{\circ} \mathrm{C}$, the crystalline alkyl tails melt, as revealed by the disappearance of the high $q$ peak at $80{ }^{\circ} \mathrm{C}$, and simultaneously, the morphology changes from lamellar to columnar hexagonal, morphology which remains stable between 80 and $120^{\circ} \mathrm{C}$. Upon further increase of the temperature up to $120^{\circ} \mathrm{C}$ or above, the order-to-disorder transition is reached, and the structure melts into a disordered isotropic fluid. Interestingly, depending on the cooling rate, both morphologies can be recovered at room temperature upon cooling from above the $\mathrm{T}_{\mathrm{ODT}}$, the hexagonal morphology being observed over the lamellar at quicker cooling rates. Quite expectedly, however, the lamellar phase with crystalline alkyl tails can be obtained from the metastable hexagonal phase at room temperature if the sample is allowed to recover for enough time (ca. 1 month). These interesting findings prove that for $\mathrm{G} 2-\mathrm{PMeDEG}_{33}-\mathrm{C}_{18} \mathrm{SO}_{4}$ : (i) at room-temperature hexagonal columnar morphologies are metastable, whereas lamellar phases are thermodynamically stable and that (ii) the presence of the tails crystallinity and the lamellar morphology are intrinsically related because both occur simultaneously. TEM pictures of both morphologies are shown in Figure 8b,c. In the first case, the hierarchical organization of lamellae is evident; in the case of Figure 8c, the hexagonal packing of the core-shell cylinders is also clearly resolved. More importantly, cylinders laying parallel to the section are also found here, revealing convincingly a white core (alkyl tails) surrounded by dark shell (dendrons). A typical cylinder on parallel projection is marked red on the upper left part of Figure 8c.

Bates et al have already shown the very close association of crystallinity and lamellar phases in poly(ethylene)poly(ethylethylene) and poly(ethylene)-poly(ethylene-propylene) diblock copolymers. ${ }^{42}$ They observe a morphological change due to crystallinity: upon cooling below the melting temperature of polyethylene, a structure with hexagonally packed cylinders transforms into a lamellar structure, again in a specific volume fraction range.
The role of crystallinity on self-assembly has also been highlighted on supramolecular complexes. Canilho et $\mathrm{al}^{43}$ have observed the clearing of the lamellar structure of surfactants ionically complexed to dendronized polymers in correspondence of the melting of crystallinity of the surfactants. A delay between the melting of the alkyl tails and the clearing of the lamellar phase has, however, been found by the same authors for hyperbranched polypeptides supramolecularly complexed to alkyl ionic tails. ${ }^{44}$ We believe that in the present case, as in ref 42 , either the uncorrelation or the simultaneous occurrence of order-order transitions and alkyl tail melting observed for G2-PMeDEG ${ }_{33}-$ $\mathrm{C}_{18} \mathrm{SO}_{4}$ and $\mathrm{G} 2-\mathrm{PMeDEG}_{26}-\mathrm{C}_{18} \mathrm{SO}_{4}$ may be related to: (i) the volume fraction of the alkyl tails themselves and (ii) to whether the hierarchical lamellar organization is the most stable morphology when the constraints imposed by the crystalline alkyl tails are released upon melting. In support of this scenario, for G2$\mathrm{PMeDEG}_{26}-\mathrm{C}_{18} \mathrm{SO}_{4}$, where the volume fraction of surfactant and dendron is 0.26 ( 0.74 polymer $)$, the lamellar structure is maintained when the crystallinity of alkyl tails melts; on the contrary, for $\mathrm{G} 2-\mathrm{PMeDEG}_{33}-\mathrm{C}_{18} \mathrm{SO}_{4}$, where the volume fraction is 0.22 ( 0.78 polymer), the stable phase upon suppression of alkyl tail crystallinity becomes the core-shell columnar hexagonal. In other terms, the boundary between hexagonally packed coreshell cylinders and hierarchically organized lamellae for this system appears to be in the region of the volume fraction of the polymer tail ranging between 0.76 and 0.78 . The crystallization of the $\mathrm{C} 18$ tails, however, further contributes to stabilizing the lamellar structure. Therefore, observing cylinders between 0.81 and 0.76 polymer tail volume fraction is not surprising, whereas the lamellar phase observed at low temperature in between this region, at 0.78 , would simply be a result of the $\mathrm{C} 18$ crystallization. This clearly demonstrates the impact of the volume fraction of tails on the properties of the supramolecular complex: the relative volume fractions can be altered, as done in the present case, by varying the length of the polymer tail emanating out of the focal point, offering an additional tunable parameter in the molecular design of the final structures. 


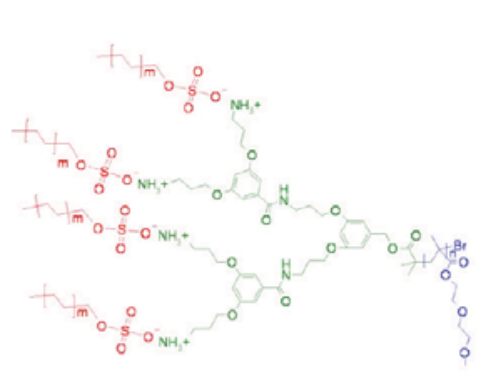

(b)

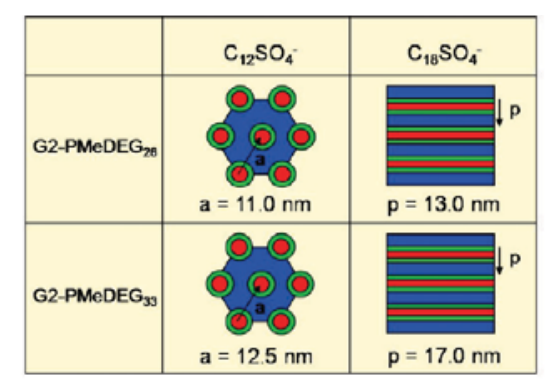

(a)

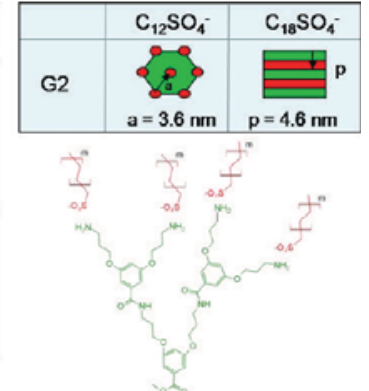

(c)

Figure 9. (a) State diagram summarizing the stable phases at room temperature for the $\mathrm{G} 2-\mathrm{PMeDEG}_{n}-\mathrm{C}_{m} \mathrm{SO}_{4}$ complexes studied. (b) Chemical structures for the complexes used in the present work. For comparison, the state diagram at room temperature for complexes based on dendron-surfactant systems identical to those studied here (but without polymer tails emanating from the focal point) is also shown in (c), ${ }^{26}$ together with the corresponding chemical structures.

The findings presented here, are further supported by available SCFTs carried out for similar dendritic pitchfork-like systems. ${ }^{28}$ In these simulations, several morphologies were found to be thermodynamically stable depending on the volume fractions of surfactants/dendrons/polymers and the degree of segregation occurring among the different constituents. In particular, coreshell columnar hexagonal phases with alkyl tails on the core of the cylinders and hierarchical lamellae analogue to those observed here, were found to be the most stable morphologies at specific volume fractions similar to those used in the present work. Furthermore, remarkable analogies can be found between the SCFT simulations and the morphologies presented here. For example, for core-shell cylinders, both experimental and SCFT simulations data agree on the compositions of all three types of microdomains, where alkyl tails form the core of the cylinders, the dendrons the shell, and the linear polymers the continuous matrix. As a further example, for the hierarchical lamellar phases, both experimental data and SCFT simulations clearly indicate that within a full lamellar lattice period, the domains formed by (a) the alkyl tails and (b) the linear polymer occur only once, whereas those formed by the dendrons occur twice.

These results not only further support the fact that the inverted and hierarchically observed morphologies are thermodynamically driven but also confirm that differently from general assumptions, ${ }^{45}$ models developed to describe phase separation for block copolymers can be adapted and used to interpret microphase separation also in dendritic systems efficiently.

Figure 9a highlights the role of the polymer chain emanating from the focal point of the dendrons by comparing the phase diagram for the second generation dendron complexed to $\mathrm{C}_{12} \mathrm{SO}_{4}{ }^{-}$or $\mathrm{C}_{18} \mathrm{SO}_{4}{ }^{-}$(Figure $\left.9 \mathrm{c}\right)^{26}$ with the corresponding dendritic pitchfork-like system (Figure 9b) and provides lattice parameters for the different structures observed. When the dendrons carry no polymer chain in the focal point, their mobility is high, and they can pack into inverted columnar hexagonal structures (with $\mathrm{C}_{12} \mathrm{SO}_{4}^{-}$) or long-range ordered lamellar phases (with $\mathrm{C}_{18} \mathrm{SO}_{4}{ }^{-}$). In both cases, the structural organization is achieved on the order of $3-5 \mathrm{~nm}$, as dictated by the liquid crystalline packing of surfactant and dendrons. In the present case, the polymer attached at the focal point of the dendrons, suppresses the organization at the liquid crystalline length scale $\left(10^{0} \mathrm{~nm}\right)$ but allows the structural packing to evolve to the polymer length scale $\left(10^{1} \mathrm{~nm}\right)$. However, the curvature at the dendron-alkyl tail interface is preserved with a core-shell morphology in the case of the hexagonal lattice and a flat interface in the case of hierarchically organized lamellae. Two factors are responsible for the inverted morphologies found in the core-shell cylinders: their low volume fraction ${ }^{46,47}$ and the favorable entropy of the short alkyl tails when these are confined within the cylinders. ${ }^{25,26}$ It is worth stressing here that whereas similar hierarchical structures (core-shell cylinders and parallel lamellae) can be found in triblock copolymer systems (although on larger length scales), ${ }^{48,49}$ their occurrence in dendritic systems has a remarkable significance because it demonstrates unambiguously that the intrinsic curvature of the dendron surface is still subject to the changes dictated by the most favorable energetic configurations in the solid state (e.g., the minimization of the free energy associated with both the entropic and the interfacial contributions).

In conclusion, we have shown that supramolecular systems with dendritic pitchfork-like structures arising from single polymer chain-terminated dendrons with alkyl tails ionically attached onto the dendron periphery, result in the self-assembly of highly ordered structures with various hierarchically organized unconventional morphologies and that these structures are found to be thermodynamically stable as per both experimental and theoretical evidence. Notably, these structures were found to consist of hierarchically packed parallel lamellae and core-shell hexagonally packed cylinders with alkyl tails confined within the cylinder cores. This makes these systems unchallenged candidates for structural hierarchical control of multidomains $(\geq 3)$ on length scales on the order of $\sim 10 \mathrm{~nm}$. The resulting materials hold great promise in the design of stimuli-responsive nanomaterials. Exploiting the supramolecular noncovalent binding between the dendrons and alkyl tails, their confinement within cylinders and the temperature-responsiveness of the linear polymer emanating from the focal point of the dendrons (the polymer used in the present system has a lower critical solubility temperature LCST of $25^{\circ} \mathrm{C}$ ) shall enable the design of new materials for templating responsive molecular filters and membranes similar to those described by Nykanen et al. ${ }^{50}$ The present systems could additionally offer a controllable pore size and lattice by simply removing the ionically bound surfactants. ${ }^{27}$ Finally, the orderorder transitions observed at moderate temperatures could also be exploited to induce sudden temperature-driven changes in materials properties, making these systems a very appealing candidate for new applications.

Acknowledgment. We are grateful to the Swiss National Foundation Proposal no. 200021-119801 for financial support.

Supporting Information Available: Detailed experimental information.

\section{References and Notes}

(1) Suárez, M.; Lehn, J. M.; Zimmerman, S. C.; Skoulios, A.; Heinrich, B. J. Am. Chem. Soc. 1998, 120, 9526-9532. 
(2) Kato, T.; Mizoshita, N.; Kishimoto, K. Angew. Chem., Int. Ed. 2006, 45, 38-68.

(3) Lehn, J. M. Science 2002, 295, 2400-2403.

(4) Kato, T. Science 2002, 295, 2414-2418.

(5) Hammond, M. R.; Mezzenga, R. Soft Matter 2008, 4, 952-961.

(6) Ikkala, O.; ten Brinke, G. Science 2002, 295, 2407-2409.

(7) Fréchet, J. M. J. Science 1994, 263, 1710-1715.

(8) Percec, V.; Ahn, C. H.; Ungar, G.; Yeardley, D. J. D.; Möller, M; Sheiko, S. S. Nature 1998, 391, 161-164

(9) Percec, V.; Cho, W. D.; Möller, M.; Prokhorova, S. A.; Ungar, G.; Yeardley, D. J. P. J. Am. Chem. Soc. 2000, 122, 4249-4250.

(10) Zeng, X. B.; Ungar, G.; Liu, Y. S.; Percec, V.; Dulcey, S. E.; Hobbs, J. K. Nature 2004, 428, 157-160.

(11) Martín-Rapún, R.; Marcos, M.; Omenat, A.; Barberá, J.; Romero, P.; Serrano, J. L. J. Am. Chem. Soc. 2005, 127, 7397-7403.

(12) Barberá, J.; Donnio, B.; Gehringer, L.; Guillon, D.; Marcos, M.; Omenat, A.; Serrano, J. L. J. Mater. Chem. 2005, 15, 4093-4105.

(13) Cho, B. K.; Jain, A.; Gruner, S. M.; Wiesner, U. Science 2004, 305, 1598-1601.

(14) Cho, B. K.; Jain, A.; Nieberle, J.; Mahajan, S.; Wiesner, U.; Gruner, S. M.; Turk, S.; Rader, H. J. Macromolecules 2004, 37, 4227-4234.

(15) Ungar, G.; Liu, Y. S.; Zeng, X. B.; Percec, V.; Cho, W. D. Science 2003, 299, 1208-1211.

(16) Percec, V.; Johansson, G.; Ungar, G.; Zhou, J. P. J. Am. Chem. Soc. 1996, 118, 9855-9866.

(17) Marcos, M.; Martín-Rapún, R.; Omenat, A.; Barberá, J.; Serrano, J. L. Chem. Mater. 2006, 18, 1206-1212.

(18) Rueff, J. M.; Barberá, J.; Donnio, B.; Guillon, D.; Marcos, M.; Serrano, J. L. Macromolecules 2003, 36, 8368-8375.

(19) Marcos, M.; Martín-Rapún, R.; Omenat, A.; Serrano, J. L. Chem. Soc. Rev. 2007, 36, 1889-1901.

(20) Cho, B. K.; Jain, A.; Mahajan, S.; Ow, H.; Gruner, S. M.; Wiesner, U. J. Am. Chem. Soc. 2004, 126, 4070-4071.

(21) Ornatska, M.; Peleshanko, S.; Genson, K. L.; Rybak, B.; Bergman, K. N.; Tsukruk, V. V. J. Am. Chem. Soc. 2004, 126, 9675-9684.

(22) Chung, Y. W.; Lee, B. I.; Kim, H. Y.; Wiesner, U.; Cho, B. K. J. Polym. Sci., Part A: Polym. Chem. 2007, 45, 4988-4994.

(23) Percec, V.; Glodde, M.; Bera, T. K.; Miura, Y.; Shiyanovskaya, I.; Singer, K. D.; Balagurusamy, V. S. K.; Heiney, P. A.; Schnell, I.; Rapp, A.; Spiess, H. W.; Hudson, S. D.; Duan, H. Nature 2002, 419, 384-387.

(24) Gehringer, L.; Guillon, D.; Donnio, B. Macromolecules 2003, 36, 5593-5601.

(25) Canilho, N.; Kasëmi, E.; Schlüter, A. D.; Ruokolainen, J.; Mezzenga, R. Macromolecules 2007, 40, 7609-7616.

(26) Mezzenga, R.; Ruokolainen, J.; Canilho, N.; Kasëmi, E.; Schlüter, A. D.; Lee, W. B.; Frederikson, G. H. Soft Matter 2009, 5, 92-97.
(27) Canilho, N.; Kasëmi, E.; Schlüter, A. D.; Ruokolainen, J.; Mezzenga, R. Macromol. Symp. 2008, 270, 58-64.

(28) Lee, W. B.; Elliott, R.; Mezzenga, R.; Fredrickson, G. H. Macromolecules 2009, 42, 849-859.

(29) Shu, L. J.; Schäfer, T.; Schlüter, A. D. Macromolecules 2000, 33, 4321-4328.

(30) Leibler, L. Macromolecules 1980, 13, 1602-1617.

(31) Mäki-Ontto, R.; de Moel, K.; de Odorico, W.; Ruokolainen, J.; Stamm, M.; ten Brinke, G.; Ikkala, O. Adv. Mater. 2001, 13, $117-121$.

(32) Ruokolainen, J.; Mäkinen, R.; Torkkeli, M.; Mäkelä, T.; Serimaa, R.; ten Brinke, G.; Ikkala, O. Science 1998, 280, 557-560.

(33) Valkama, S.; Kosonen, H.; Ruokolainen, J.; Haatainen, T.; Torkkeli, M.; Serimaa, R.; ten Brinke, G.; Ikkala, O. Nat. Mater. 2004, 3, 872-876.

(34) Gobius du Sart, G.; Vukovic, I.; van Ekenstein, G. A.; Polushkin, E.; Loos, K.; ten Brinke, G. Macromolecules 2010, 43, 2970-2980.

(35) Li, C.; Schlüter, A. D.; Zhang, A.; Mezzenga, R. Adv. Mater. 2008, $20,4530-4534$

(36) Nagata, Y.; Masuda, J.; Noro, A.; Cho, D.; Takano, A.; Matsushita, Y. Macromolecules 2005, 38, 10220-10225.

(37) Masuda, J.; Takano, A.; Nagata, Y.; Noro, A.; Matsushita, Y. Phys. Rev. Lett. 2006, 1997, 098301.

(38) Matsushita, Y.; Takano, A.; Hayashida, K.; Asari, T.; Noro, A. Polymer 2009, 50, 2191-2203.

(39) Klymko, T.; Markov, V.; Subbotin, A.; ten Brinke, G. Soft Matter 2009, 5, 98-103.

(40) Fleury, G.; Bates, F. S. Macromolecules 2009, 42, 1691-1694.

(41) Fleury, G.; Bates, F. S. Macromolecules 2009, 42, 3598-3610.

(42) Ryan, A. J.; Hamley, I. W.; Bras, W.; Bates, F. S. Macromolecules 1995, 28, 3860-3868

(43) Canilho, N.; Kasëmi, E.; Schlüter, A. D.; Mezzenga, R. Macromolecules 2007, 40, 2822-2830.

(44) Canilho, N.; Scholl, M.; Klok, H. A.; Mezzenga, R. Macromolecules 2007, 40, 8374-8383.

(45) Rosen, B. M.; Wilson, C. J.; Wilson, D. A.; Peterca, M.; Imam, M. R.; Percec, V. Chem. Rev. 2009, 109, 6275-6540.

(46) Fuchs, P.; Tschierske, C.; Raith, K.; Das, M. K.; Diele, S. Angew. Chem., Int. Ed. 2002, 41, 628-631.

(47) Cheng, X. H.; Das, M. K.; Diele, S.; Tschierske, C. Langmuir 2002, $18,6521-6529$

(48) Hückstädt, H.; Göpfert, A.; Abetz, V. Polymer 2000, 41, 9089 9094.

(49) Bailey, T. S.; Pham, H. D.; Bates, F. S. Macromolecules 2001, 34, 6994-7008.

(50) Nykänen, A.; Nuopponen, M.; Laukkanen, A.; Rytelä, M.; Turunen, O.; Tenhu, H.; Mezzenga, R.; Ikkala, O.; Ruokolainen, J. Macromolecules 2007, 40, 5827-5834. 